\title{
Orbits and masses in the multiple system LHS $1070^{\star}$
}

\author{
R. Köhler ${ }^{1,2}$, T. Ratzka ${ }^{3}$, and Ch. Leinert ${ }^{1}$ \\ ${ }^{1}$ Max-Planck-Institut für Astronomie, Königstuhl 17, 69117 Heidelberg, Germany \\ e-mail: koehler@mpia.de \\ 2 Landessternwarte, Zentrum für Astronomie der Universität Heidelberg, Königstuhl, 69117 Heidelberg, Germany \\ e-mail: r.koehler@lsw.uni-heidelberg.de \\ ${ }^{3}$ Universitäts-Sternwarte München, Ludwig-Maximilians-Universität, Scheinerstr. 1, 81679 München, Germany
}

Received 22 December 2011 / Accepted 15 March 2012

\section{ABSTRACT}

\begin{abstract}
Aims. We present a study of the orbits of the triple system LHS 1070, with the aim to determine individual masses of its components. Methods. Sixteen new relative astrometric positions of the three components in the K band were obtained with NACO at the VLT, Omega CASS at the $3.5 \mathrm{~m}$ telescope on Calar Alto, and other high-spatial-resolution instruments. We combined them with data from the literature and fit orbit models to the dataset. We derived an improved fit for the orbit of LHS $1070 \mathrm{~B}$ and C around each other, and an estimate for the orbit of $\mathrm{B}$ and $\mathrm{C}$ around $\mathrm{A}$.

Results. The orbits are nearly coplanar, with a misalignment angle of less than $10^{\circ}$. The masses of the three components are $M_{A}=$ $0.13 \ldots 0.16 M_{\odot}, M_{B}=0.077 \pm 0.005 M_{\odot}$, and $M_{C}=0.071 \pm 0.004 M_{\odot}$. Therefore, LHS $1070 \mathrm{C}$ is certainly, and LHS $1070 \mathrm{~B}$ probably a brown dwarf. Comparison with theoretical isochrones shows that LHS $1070 \mathrm{~A}$ is either fainter or more massive than expected. One possible explanation would be that it is a binary. However, the close companion reported previously could not be confirmed.
\end{abstract}

Key words. stars: low-mass - brown dwarfs - stars: fundamental parameters - stars: individual: LHS 1070 - binaries: close celestial mechanics

\section{Introduction}

LHS 1070 (other common names are GJ 2005, LP 881-64, 2MASS J00244419-2708242) is a nearby high-proper-motion star located in the south galactic pole region. It was observed by Leinert et al. (1994) as part of their near-infrared speckle survey for duplicity of nearby southern $\mathrm{M}$ dwarves. The group detected in 1993 two companions located 1.1" and 1.3" north of the primary by using the SHARP camera mounted at the NTT (La Silla, Chile) and applying Speckle imaging. In a consecutive measurement the companions exhibited an orbital motion around each other and around the primary while following the proper motion of the system. A fourth component $\mathrm{D}$ was identified by Henry et al. (1999) with the fine guidance sensors onboard the Hubble Space Telescope (HST). A separation of only about 50 mas from component A was reported. Speckle interferometric and adaptive optics measurements, however, were not able to confirm LHS 1070 D (Leinert et al. 2001; Seifahrt et al. 2008). This might be due to the small separation, but it is also possible that the detection is spurious (Henry, priv. comm.).

When comparing the colors of the components, the companions appear redder than the primary (Leinert et al. 1994). First estimates already identified the two companions as stars close to the hydrogen burning limit that divides brown dwarves from main-sequence stars. This mass range is characterised by a strong decrease of the effective temperature and the onset of dust formation in the atmospheres. Photometric and spectroscopic measurements with the HST in the visual indeed were

* Based on observations collected at the European Southern Observatory, Chile, proposals number 60.A-9026, 66.C-0219, 67.C-0354, 68.C-0539, 70.C-0476, 072.C-0022, 074.C-0637, 078.C-0386, 380.C-0179, 382.C-0324, and 382.C-0329. represented by model atmospheres containing dust (Leinert et al. 2000). Also the low masses could be confirmed. The spectral classifications of the companions are M8.5V and M9-9.5V. The primary has an earlier spectral type of M5.5-6V.

Reiners et al. (2007) found for the components B and C a similarily high rotational velocity of $v \sin i=16 \mathrm{~km} \mathrm{~s}^{-1}$, which is twice that of the primary. The authors conclude that the temperature-dependent magnetic braking was acting for $1 \mathrm{Gyr}$ on all three components. The main difference between the otherwise very similar components $\mathrm{B}$ and $\mathrm{C}$ is the higher activity of component $\mathrm{B}$. This might be related to the higher temperature or the higher magnetic flux. In the HST spectra $\mathrm{H} \alpha$ emission was found towards LHS $1070 \mathrm{~A}$ and B, but not towards component C (Leinert et al. 2000). Recently, photometric activity of the two more massive stars was identified (Almeida et al. 2011). Component B showed a brightness increase in the visual that is among the largest ever observed in a flare star.

The very low mass companions of LHS 1070 provide the possibility to refine the models of (pre-)main-sequence stars close to the transition region to brown dwarves. It is thus very important to derive the exact masses of these stars. The small separation of the components B and C, i.e. their short orbital period is very well suited to allow even for this low mass stars a mass determination by fitting the orbital elements. The dynamical mass has the advantage of being independent from theoretical models. It is thus a precious probe to test evolutionary models.

The main uncertainty for the mass determination is the distance to the object. The trigonometric parallax of LHS 1070 derived by van Altena et al. (1995) is $135.3 \pm 12.1$ mas. This value was later refined to $129.47 \pm 2.48$ mas, placing the system at a distance of $7.72 \pm 0.15 \mathrm{pc}$ (Costa et al. 2005). The proper motion of LHS 1070 is $653.7 \pm 0.3 \mathrm{mas} / \mathrm{yr}$, corresponding to $23.9 \mathrm{~km} \mathrm{~s}^{-1}$, 
and directed along the postion angle $348.3^{\circ} \pm 0.44^{\circ}$. In addition, radial velocity measurements found that LHS 1070 is approaching with $36.4 \mathrm{~km} \mathrm{~s}^{-1}$ to the Sun (Basri \& Marcy 1995). Due to its kinematic properties, LHS 1070 can be associated with the old disk population.

A first successful fit of the close orbit of component $\mathrm{C}$ around component B was presented by Leinert et al. (2001). Based on Speckle interferometric measurements a semi-major axis of $a=446 \pm 29$ mas and a period of $P=16.1 \pm 1.4 \mathrm{yr}$ were derived. When taking the refined distance of Costa et al. (2005) into account, the corresponding dynamical mass is $0.157 \pm 0.042 M_{\odot}$. A consistent mass of $0.157 \pm 0.009 M_{\odot}$ was found by Seifahrt et al. (2008), who derived a wider ( $a=461.9 \pm 0.7$ mas $)$ orbit, but with $P=17.0$ yr. Due to the better coverage the authors presented also a first reliable fit of the wide orbit of the components $\mathrm{B}$ and $\mathrm{C}$ around the primary. The dynamical mass of the whole system was determined as $0.272 \pm 0.017 M_{\odot}$. The orbits were found to be coplanar.

In this study, we present new measurements taken both with Speckle interferometric techniques and adaptive optics in the $K$-band (Sect. 2). The data is carefully selected and calibrated on a case by case basis with the aim to reach the highest degree of consistency possible. These new data are not only used to further refine the fits of the close (Sect. 3) and the wide orbit (Sect. 4). We utilise the primary component as astrometric reference, which enables the determination of the mass ratio of the components $\mathrm{B}$ and $\mathrm{C}$. With this knowledge the individual masses of all three components can be derived. In Sect. 5 we discuss the implications of our findings with respect to the stability of the system and its evolutionary stage. We conclude in Sect. 6.

\section{Observations and data reduction}

Our group monitored LHS 1070 since the discovery of its two companions, using a number of different telescopes and instruments. Table 1 gives a journal of observations and the measured relative positions. The following subsections describe the individual instruments and data reduction procedures.

\subsection{ESO NTT/SHARP}

The SHARP I camera (System for High Angular Resolution Pictures) of the Max-Planck-Institut for Extraterrestrial Physics (Hofmann et al. 1992) was already used for many of the observations presented in Leinert et al. (2001). We used it again during an observing campaign in June and July 2001 at the European Southern Observatory (ESO) $3.5 \mathrm{~m}$ New Technology Telescope (NTT) on La Silla, Chile. LHS 1070 was observed in the $K$-band at $2.2 \mu \mathrm{m}$ in four different nights. SHARP is a camera for speckle interferometry, which means that one observation consists of several hundred frames with short integration times $(0.5 \mathrm{~s})$. To reduce the data, we used our speckle program (Köhler et al. 2000). The same observing and data reduction strategy was already employed for the data published in Leinert et al. (2001) and a number of other multiplicity studies.

\subsection{ESO 3.6 m/ADONIS/SHARP II}

In January and December 2001, LHS 1070 was observed with the adaptive optics (AO) system ADONIS (Rousset \& Beuzit 1999) and the SHARP II camera (Hofmann et al. 1995) at the ESO $3.6 \mathrm{~m}$ telescope on La Silla, Chile. We used the $K$-band filter of this instrument, which has a central wavelength of
$2.177 \mu \mathrm{m}$. The observing strategy was "AO-assisted speckle interferometry", meaning we took many frames with short integration times. In January, we recorded 1000 frames with an integration time of $0.5 \mathrm{~s}$ each. In December, we took 240 frames with a longer integration time of $3 \mathrm{~s}$ each. For the data reduction, we used the same programs and algorithms as for the speckle data.

\subsection{Calar Alto $3.5 \mathrm{~m} /$ Omega Cass}

On several occasions between 2000 and 2006, LHS 1070 was observed with the Omega Cass camera (Lenzen et al. 1998) at the $3.5 \mathrm{~m}$-telescope on Calar Alto, Spain. The camera is equipped with a $1024 \times 1024$-pixel detector, but we used only a subarray of $128 \times 128$ pixels to enable the fast read-out mode required for Speckle-interferometry. The observations were done in the $K$-band and with the highest resolution optics of the instrument (pixel scale $\sim 95$ mas/pixel). Our speckle program was used again to reduce the data.

\subsection{ESO VLT/NACO}

The majority of new observations were taken with NAOS/CONICA (NACO for short), the adaptive optics, near-infrared camera at the ESO Very Large Telescope (VLT) on Cerro Paranal, Chile (Rousset et al. 2003; Lenzen et al. 2003). LHS 1070 was observed in the course of several programs (see Table 1). To ensure a consistent data set, only imaging observations in the $K_{\mathrm{s}}$ photometric band were used for the orbit determination.

The NACO images were sky subtracted with a median sky image, and bad pixels were replaced by the median of the closest good neighbors. Finally, the images were visually inspected for any artifacts or residuals. Figure 1 shows an example of the results. The Starfinder program (Diolaiti et al. 2000) was used to measure the positions of the stars. The positions in several images taken during one observation were averaged, and their standard deviation used to estimate the errors.

\subsection{Plate scale and orientation}

For a heterogenous data set like this, it is crucial to calibrate the absolute pixel scale and orientation of each observation. To this end, we took images of fields in the Orion Trapezium during each observing campaign, and reduced them in the same way as the images of the science targets. The measured positions of the cluster stars were compared with the coordinates given in McCaughrean \& Stauffer (1994). The mean pixel scale and orientation were computed from a global fit of all star positions. The scatter of values derived from star pairs were used to estimate the errors (see Sect. 2.6).

The errors of the calibration are usually comparable to or larger than the errors of the measured positions of the science target, indicating the importance of a proper astrometric calibration. For this reason, we decided to use in this work only data where images of the Orion Trapezium were taken within a few days. We have two observations of LHS 1070 that were taken in June and July, when Orion was not observable. These observations are not used here, in order to ensure a consistent calibration of all the data. For the same reason, we do no use data from the literature that could not be re-calibrated by our group. 

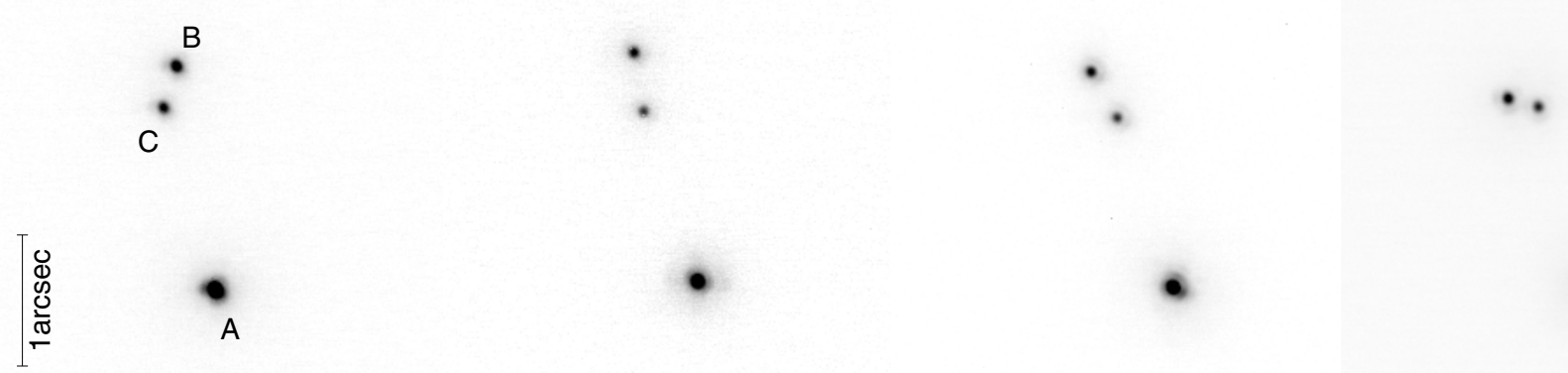

Fig. 1. Images of LHS 1070 obtained with NACO in December 2002, December 2004, October 2006, and November 2008. North is up, and East to the left. The separation between the two components B and C changes from about 330 mas in 2002 to about 240 mas in 2008 (cf. Table 1).

Table 1. Astrometric measurements of LHS 1070 B-C.

\begin{tabular}{|c|c|c|c|c|c|}
\hline \multirow[t]{2}{*}{ Date (UT) } & \multicolumn{2}{|c|}{ A-B } & \multicolumn{2}{|c|}{$\mathrm{B}-\mathrm{C}$} & \multirow[t]{2}{*}{ Reference, instrument, proposal-No } \\
\hline & $d$ [mas] & $\mathrm{PA}\left[{ }^{\circ}\right]$ & $d$ [mas] & $\mathrm{PA}\left[^{\circ}\right]$ & \\
\hline 1993 Jul. 29 & $1072 \pm 10$ & $-8.6 \pm 0.3$ & 266. \pm 5 & $328.5 \pm 0.7$ & Leinert et al. (2001) \\
\hline 1994 May 1 & $1085 \pm 4$ & $-7.7 \pm 0.1$ & 341. \pm 5 & $346.9 \pm 0.6$ & Leinert et al. (2001) \\
\hline 1994 Sep. 15 & $1092 \pm 9$ & $-7.2 \pm 0.4$ & 375. \pm 4 & $352.7 \pm 0.61$ & Leinert et al. (2001) \\
\hline 1994 Sep. 24 & $1095 \pm 12$ & $-6.8 \pm 0.42$ & 382. \pm 5 & $353.9 \pm 0.67$ & Leinert et al. (2001) \\
\hline 1995 Jan. 17 & $1094 \pm 6$ & $-6.2 \pm 0.3$ & 400. \pm 8 & $356.1 \pm 0.4$ & Leinert et al. (2001) \\
\hline 1995 Jul. 9 & $1119 \pm 11$ & $-6.4 \pm 0.1$ & 439. \pm 4 & $3.2 \pm 0.1$ & Leinert et al. (2001) \\
\hline 1995 Jul. 14 & $1102 \pm 3$ & $-6.1 \pm 0.1$ & 436. \pm 1 & $3.7 \pm 0.2$ & Leinert et al. (2001) \\
\hline 1996 Jan. 16 & $1124 \pm 2$ & $-5.6 \pm 0.3$ & 459. \pm 3 & $9.0 \pm 0.3$ & Leinert et al. (2001) \\
\hline 1996 Aug. 22 & $1161 \pm 5$ & $-5.0 \pm 0.1$ & 465. \pm 3 & $14.9 \pm 0.1$ & Leinert et al. (2001) \\
\hline 1996 Sep. 27 & $1157 \pm 8$ & $-4.7 \pm 0.1$ & 468. \pm 4 & $15.8 \pm 0.1$ & Leinert et al. (2001) \\
\hline 1997 Jul. 15 & $1235 \pm 14$ & $-3.5 \pm 0.23$ & 458. \pm 6 & $23.4 \pm 0.6$ & Leinert et al. (2001) \\
\hline 1997 Aug. 25 & $1243 \pm 7$ & $-3.5 \pm 0.23$ & 450. \pm 15 . & $25.4 \pm 1.0$ & Leinert et al. (2001) \\
\hline 1997 Nov. 17 & $1223 \pm 5$ & $-2.9 \pm 0.1$ & 439. \pm 8 & $26.5 \pm 0.45$ & Leinert et al. (2001) \\
\hline 1998 Jan. 2 & $1260 \pm 3$ & $-2.5 \pm 0.14$ & 432. \pm 3 & $28.0 \pm 0.45$ & Leinert et al. (2001) \\
\hline 1998 May 7 & $1281 \pm 7$ & $-1.9 \pm 0.1$ & 408. \pm 8 & $32.3 \pm 0.73$ & Leinert et al. (2001) \\
\hline 1998 Oct. 10 & $1332 \pm 8$ & $-1.0 \pm 0.1$ & 377. \pm 19 . & $38.2 \pm 0.5$ & Leinert et al. (2001) \\
\hline 1999 Jun. 18 & $1404 \pm 3$ & $0.44 \pm 0.1$ & 318. \pm 2 & $49.4 \pm 0.4$ & Leinert et al. (2001) \\
\hline 1999 Aug. 3 & $1407 \pm 7$ & $1.02 \pm 0.28$ & 303. \pm 7 & $52.1 \pm 0.3$ & Leinert et al. (2001) \\
\hline 1999 Sep. 1 & $1414 \pm 4$ & $1.23 \pm 0.17$ & 292. \pm 8 & $54.6 \pm 1.0$ & Leinert et al. (2001) \\
\hline 1999 Nov. 23 & $1437 \pm 4$ & $1.64 \pm 0.11$ & 279. \pm 5 & $60.1 \pm 0.9$ & Leinert et al. (2001) \\
\hline 2000 Jun. 4 & $1487 \pm 9$ & $2.5 \pm 0.2$ & $240 . \pm 2$ & $76.0 \pm 0.4$ & Leinert et al. (2001) \\
\hline 2000 Jun. 20 & $1518 \pm 3$ & $3.6 \pm 0.3$ & 237. \pm 3 & $80.8 \pm 0.7$ & Leinert et al. (2001) \\
\hline 2000 Oct. $31-$ Nov. 1 & $1516 \pm 1.4$ & $4.4 \pm 0.1$ & $218.1 \pm 1.9$ & $88.9 \pm 0.6$ & OCASS \\
\hline 2001 Jan. 9 & $1546.4 \pm 1.1$ & $4.7 \pm 0.1$ & $219.0 \pm 9.0$ & $100.4 \pm 2.3$ & ADONIS, 66.C-0219 \\
\hline 2001 Jun. 29-Jul. 6 & $1600.7 \pm 5.5$ & $5.4 \pm 0.1$ & $222.7 \pm 1.0$ & $120.3 \pm 0.2$ & SHARP, 67.C-0354 \\
\hline 2001 Dec. 6 & $1631.2 \pm 2.5$ & $6.8 \pm 0.2$ & $244.9 \pm 0.4$ & $136.1 \pm 0.2$ & NACO, 60.A-9026 (commissioning) \\
\hline 2001 Dec. 11 & $1633.0 \pm 7.7$ & $7.1 \pm 0.4$ & $246.7 \pm 3.8$ & $136.8 \pm 0.9$ & ADONIS, 68.C-0539 \\
\hline 2002 Dec. 16 & $1712.1 \pm 3.4$ & $9.8 \pm 0.3$ & $327.7 \pm 0.9$ & $162.8 \pm 0.3$ & NACO, 70.C-0476 \\
\hline 2003 Dec. 12 & $1772.3 \pm 4.9$ & $12.7 \pm 0.1$ & $406.3 \pm 1.1$ & $178.2 \pm 0.1$ & NACO, 072.C-0022 \\
\hline 2004 Dec. 11 & $1796.3 \pm 2.2$ & $15.9 \pm 0.1$ & $450.0 \pm 1.4$ & $189.7 \pm 0.2$ & NACO, 074.C-0637 \\
\hline 2005 Sep. 25 & 1792. \pm 19 & $17.9 \pm 0.2$ & 450. \pm 11 & $198.7 \pm 0.9$ & OCASS \\
\hline 2006 Sep. $1-2$ & $1759.2 \pm 4.6$ & $20.5 \pm 0.2$ & $403.1 \pm 6.0$ & $208.1 \pm 0.9$ & OCASS \\
\hline 2006 Oct. 30 & $1742.9 \pm 2.9$ & $20.9 \pm 0.5$ & $399.7 \pm 1.0$ & $209.6 \pm 0.5$ & NACO, 078.C-0386 \\
\hline 2006 Nov. 28 & $1744.7 \pm 3.8$ & $21.3 \pm 0.2$ & $396.1 \pm 2.0$ & $211.0 \pm 0.3$ & OCASS \\
\hline 2007 Sep. 16 & $1680.2 \pm 1.5$ & $23.6 \pm 0.2$ & $328.7 \pm 0.4$ & $223.5 \pm 0.2$ & NACO, 380.C-0179 \\
\hline 2008 Feb. 1 & $1646.4 \pm 1.4$ & $24.8 \pm 0.2$ & $295.4 \pm 0.4$ & $231.5 \pm 0.2$ & NACO, 380.C-0179 \\
\hline 2008 Oct. 17,18 & $1576.6 \pm 0.7$ & $27.3 \pm 0.1$ & $240.7 \pm 0.3$ & $252.6 \pm 0.13$ & NACO, 382.C-0324/0329 \\
\hline 2008 Nov. 6 & $1571.3 \pm 0.7$ & $27.4 \pm 0.1$ & $237.6 \pm 0.3$ & $254.6 \pm 0.12$ & NACO, 382.C-0324 \\
\hline
\end{tabular}

\subsection{Astrometric error estimates}

Independent of instrument and telescope used, our observation resulted in at least four images or speckle cubes for each epoch. The final result was obtained by averaging the relative positions measured in individual images. As estimate for the errors, we use the standard deviation.
The error of the astrometric calibration was estimated in a similar way. We computed the pixel scale by dividing the separation of star pairs in arcseconds by the measured separation in pixels. Similarly, the orientation is the difference of the position angle on the sky and on the detector. The standard deviation of the results for the many star pairs in our calibration field was used as estimate for the error. 
Table 2. Parameters of the best orbital solution for the pair B-C.

\begin{tabular}{|c|c|c|}
\hline \multirow{2}{*}{$\begin{array}{l}\text { Orbital element } \\
\text { Date of periastron } T_{0}\end{array}$} & \multicolumn{2}{|c|}{ Value } \\
\hline & \multicolumn{2}{|c|}{$\begin{array}{c}2454145.0_{-0.8}^{+1.4} \\
\quad(2007 \text { Feb. 13) }\end{array}$} \\
\hline Period $P$ (years) & 17.24 & ${ }_{-0.01}^{+0.01}$ \\
\hline Semi-major axis $a$ (mas) & 457.8 & $\begin{array}{l}+0.3 \\
-0.4\end{array}$ \\
\hline Semi-major axis $a(\mathrm{AU})$ & 3.53 & $\begin{array}{l}+0.07 \\
-0.07\end{array}$ \\
\hline Eccentricity $e$ & 0.0227 & ${ }_{-0.0003}^{+0.0004}$ \\
\hline Argument of periastron $\omega\left(^{\circ}\right)$ & 217.01 & $\begin{array}{l}+0.04 \\
-0.08\end{array}$ \\
\hline PA of ascending node $\Omega\left(^{\circ}\right)$ & 14.65 & $\begin{array}{l}+0.04 \\
-0.06\end{array}$ \\
\hline Inclination $i\left(^{\circ}\right)$ & 61.82 & $\begin{array}{l}+0.04 \\
-0.04\end{array}$ \\
\hline System mass $M_{\mathrm{S}}\left(\mathrm{mas}^{3} /\right.$ year $\left.^{2}\right)$ & $(3.23 \pm$ & $0.01) \times 10^{5}$ \\
\hline System mass $M_{B}+M_{C}\left(M_{\odot}\right)$ & 0.149 & \pm 0.009 \\
\hline \multicolumn{3}{|l|}{ Mean absolute difference } \\
\hline observed-predicted (mas) & 4.9 & \\
\hline Reduced $\chi^{2}$ & 3.5 & \\
\hline
\end{tabular}

An additional source of error might be the motion of the telescope between the observation of the calibration field and the target. This should not cause much uncertainty for telescopes on a parallactic mount (i.e. the Calar Alto $3.5 \mathrm{~m}$ and the ESO $3.6 \mathrm{~m}$ ). However, telescopes on an alt-azimuth mount (ESO NTT and VLT) have to correct for image rotation, which might introduce an error on the order of $0.1^{\circ}$ (W. Brandner, priv. comm.). Additionally, the opening and closing of the AO-loop, in connection with the active optics of the primary mirror, might induce small changes in the image scale. Our calibrations show that there are small changes in pixel scale and orientation on the time scale of weeks or months, but - to the best of our knowledge - there has been no study of short-term variations.

This unknown error source might explain why we seem to underestimate our errors, which results in orbit fits with $\chi^{2}>1$ (cf. Tables 2 and 3 ).

\section{The orbit of LHS $1070 \mathrm{~B}-\mathrm{C}$}

We estimated the orbital parameters of the B-C pair by fitting orbit models to all observations listed in Table 1. We followed the procedure described in Köhler et al. (2008): a grid-search in eccentricity $e$, period $P$, and time of periastron $T_{0}$. At each grid point, the Thiele-Innes elements were determined by a linear fit to the observational data using Singular Value Decomposition. From the Thiele-Innes elements, the semimajor axis $a$, the angle between node and periastron $\omega$, the position angle of the line of nodes $\Omega$, and the inclination $i$ were computed.

Since the orbit of LHS 1070 B-C is already quite wellknown (Leinert et al. 2001; Seifahrt et al. 2008), only a small range of parameter values had to be scanned: 200 points within $0.018 \leq e \leq 0.028,200$ points within $17.21 \mathrm{yr} \leq P<17.31 \mathrm{yr}$, and initially 200 points for $T_{0}$ distributed over one orbital period. After the initial scan over $T_{0}$, the best estimate for $T_{0}$ was improved by re-scanning a narrower range in $T_{0}$ centered on the minimum found in the coarser scan. This grid refinement was repeated until the step size was less than one day.

We improved the results of the grid-search with a LevenbergMarquardt $\chi^{2}$ minimization algorithm (Press et al. 1992) that fits for all 7 parameters simultaneously. The simple-minded approach would be to use the orbital elements with the minimum $\chi^{2}$ found with the grid-search. However, initial test runs showed

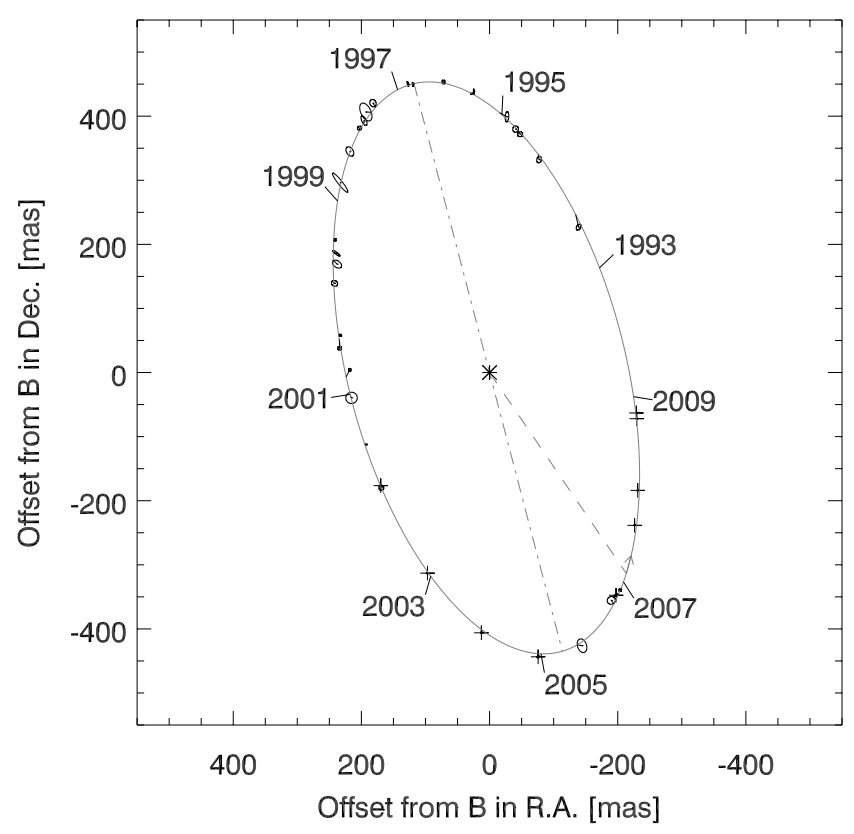

Fig. 2. The orbit of component $\mathrm{C}$ around component $\mathrm{B}$. The observed positions are marked by their error ellipses and lines connecting the observed and calculated position at the time of the observations. The observations with NACO are marked by crosses. Their errors are too small to be discernible. The dash-dotted line indicates the line of nodes, the dashed line the periastron, and the arrow shows the direction of the orbital motion.

that the algorithm does not converge on the global minimum. For the same reason, we did not use one of the previously published orbit solutions as starting point (Leinert et al. 2001; Seifahrt et al. 2008). To make sure we find the globally minimum $\chi^{2}$, we decided to use all orbits resulting from the grid-search as starting points that had $\chi^{2}<\chi_{\min }^{2}+9$. The number 9 was chosen arbitrarily, to avoid starting from obviously bad orbits. The orbit with the globally minimum $\chi^{2}$ found in by the Levenberg-Marquardt fit is shown in Fig. 2, and its elements are listed in Table 2. To convert the semi-major axis from mas to AU, we used the distance of $7.72 \pm 0.15 \mathrm{pc}$ (Costa et al. 2005).

The reduced $\chi^{2}$ of 3.5 is higher than expected for a good fit, which indicates that we underestimate our astrometric errors. The mean absolute difference between observed and predicted positions is also larger than typical errors of our measurements. This might be caused by unknown errors in the calibration, as mentioned in Sect. 2.6. However, the residuals show no pattern indicating a systematic error, and the errors given in Table 2 are our best guess. Therefore, we decided to accept the fit as it is.

Errors of the orbital elements were determined by studying the $\chi^{2}$ function around its minimum. Since we are interested in the confidence interval for each parameter taken separately, we have to perturb one parameter (for example $T_{0}$ ) away from the minimum, and optimize all the other parameters. Any perturbation of a parameter will of course lead to a larger $\chi^{2}$. The range in $T_{0}$ within which $\chi^{2}\left(T_{0}\right)-\chi_{\min }^{2}<1$ defines the $68 \%$ confidence interval for $T_{0}$. This interval is usually not symmetric around the $T_{0}$ of the best fit, therefore we list in Table 2 separate limits for positive and negative perturbations. It should be noted that these limits describe the parameter range that contain $68 \%$ of the probability distribution, which is equivalent to the commonly used $1 \sigma$-errors. However, the errors are not normally distributed, therefore a $2 \sigma$ interval will not contain $95 \%$ of the probability distribution. 
Table 3. Parameters of the best solution for the orbit of $\mathrm{BC}$ around $\mathrm{A}$.

\begin{tabular}{lcl}
\hline \hline Orbital element & \multicolumn{2}{c}{ Value } \\
\hline Date of periastron $T_{0}$ & $2459727.9_{-3.1}^{+3.6}$ \\
& $(2022$ May 28$)$ \\
Period $P$ (years) & 44.4 & ${ }_{-2.4}^{+11.9}$ \\
Semi-major axis $a$ (mas) & 1111.6 & ${ }_{-1.1}^{+0.8}$ \\
Semi-major axis $a(\mathrm{AU})$ & 8.58 & ${ }_{-0.17}^{+0.17}$ \\
Eccentricity $e$ & 0.5200 & ${ }_{-0.0012}^{+0.0020}$ \\
Argument of periastron $\omega\left(^{\circ}\right)$ & 147.61 & ${ }_{-0.55}^{+7.94}$ \\
PA of ascending node $\Omega\left(^{\circ}\right)$ & 26.80 & ${ }_{-0.08}^{+0.08}$ \\
Inclination $i\left(^{\circ}\right)$ & 54.75 & ${ }_{-0.10}^{+0.90}$ \\
System mass $M_{A}+M_{B}+M_{C}\left(\mathrm{mas}^{3} / \mathrm{yr}^{2}\right)$ & $(6.98 \pm$ & $0.02) \times 10^{5}$ \\
System mass $M_{A}+M_{B}+M_{C}\left(M_{\odot}\right)$ & 0.321 & ${ }_{-0.028}^{+0.020}$ \\
Mass ratio $M_{\mathrm{C}} / M_{\mathrm{B}}$ & 0.92 & \pm 0.01 \\
Mean absolute difference & & \\
$\quad$ observed-predicted $(\mathrm{mas})$ & 7.9 & \\
Reduced $\chi^{2}$ & 2.6 & \\
\hline Mass of A $M_{\mathrm{A}}\left(M_{\odot}\right)$ & 0.172 & \pm 0.010 \\
Mass of B $M_{\mathrm{B}}\left(M_{\odot}\right)$ & 0.077 & \pm 0.005 \\
Mass of C $M_{\mathrm{C}}\left(M_{\odot}\right)$ & 0.071 & \pm 0.004 \\
\hline
\end{tabular}

Estimating the error of the mass required a special procedure. The mass itself is computed using Kepler's third law $\left(M=a^{3} / P^{2}\right)$. The semi-major axis $a$ and the period $P$ are usually strongly correlated. To obtain a realistic estimate for the mass error, we did not use the naive way of error propagation. Instead, we considered a set of orbital elements where the semi-major axis was replaced by the mass. This is possible because Kepler's third law gives an unambiguous relation between the two sets of elements. With the mass being one of the orbital elements, we can treat it as one of the independent fit parameters and determine its error with the method described in the previous paragraph.

With an orbit derived from astrometric measurements, there always remains the ambiguity which of the two nodes is the ascending node (defined as the node where the companion is receding from the observer). Fortunately, Seifahrt et al. (2008) measured the relative radial velocities of LHS 1070 B and C on 2006 October 9. They found it to be negative, i.e. LHS $1070 \mathrm{C}$ was approaching $\mathrm{us}^{1}$. Therefore, the position angle of the ascending node is $14.65^{\circ}$ (cf. Fig. 2), and the position angle of the descending node is $194.65^{\circ}$. Our orbit model predicts a relative radial velocity of $-4.8 \pm 0.1 \mathrm{~km} \mathrm{~s}^{-1}$ on 2006 Oct. 9 , in agreement with the measurement of Seifahrt et al. (2008).

\section{The orbit of LHS $1070 \mathrm{BC}$ around $\mathrm{A}$}

The orbit of components $\mathrm{B}$ and $\mathrm{C}$ around each other allows us to determine only the combined mass of B and C. To compute the individual masses, we need to know the mass ratio $q$, which can be computed if the position of the center of mass (CM) of $B$ and $\mathrm{C}$ is known. Unfortunately, we cannot observe the CM directly. However, we know that the $\mathrm{CM}$ of $\mathrm{B}$ and $\mathrm{C}$ is in orbit around

\footnotetext{
1 It is not entirely clear whether Seifahrt et al. (2008) give the velocity of $\mathrm{C}$ minus $\mathrm{B}$, but they report $\Omega=14.5^{\circ}$, which is consistent with our interpretation
}

component $\mathrm{A}^{2}$, and that $\mathrm{B}$ and $\mathrm{C}$ are in orbit around their $\mathrm{CM}$. The $\mathrm{CM}$ is always on the line between $\mathrm{B}$ and $\mathrm{C}$, and its distance from $\mathrm{B}$ is the constant fraction $q /(1+q)$ of the separation of $\mathrm{B}$ and $\mathrm{C}$.

We follow the method that was used by Köhler et al. (2008) to derive masses in the triple system $\mathrm{T}$ Tauri. The position of the $\mathrm{CM}$ of $\mathrm{B}$ and $\mathrm{C}$ is described in two ways: first, it is on a Keplerian orbit around A, which is described by 7 orbital elements. Second, the position of the CM can be computed from the observed positions of $\mathrm{B}$ and $\mathrm{C}$, and the mass ratio (which is treated as a free parameter). Standard error propagation is used to obtain an error estimate for this position. To compute $\chi^{2}$, we compare the position of the $\mathrm{CM}$ from the orbit around A with the positions derived from the observations. Our model has therefore 8 free parameters, the 7 elements which describe the orbit of the $\mathrm{CM}$ of $\mathrm{B}+\mathrm{C}$ around $\mathrm{A}$, and the parameter $f=q /(1+q)$. The parameter $f$ is often called fractional mass (Heintz 1978), since it is the secondary star's fraction of the total mass in a binary. It is useful in our case because it also describes the fractional offset of the CM from B, i.e. the separation between B and the CM divided by the separation between B and C. For a grid-search, $f$ is better suited than $q$, because $f$ is confined to the range 0 to 1 , while $q$ is a number between 0 and infinity.

The fitting procedure is similar to that used for the orbit of B-C, except that the grid-search is carried out in 4 dimensions: eccentricity $e$, period $P$, time of periastron $T_{0}$, and the fractional mass $f$. Singular Value Decomposition was used to fit the Thiele-Innes constants, which give the remaining orbital elements. It is worth noting that the orbital elements in this fit describe the orbit of the A-BC binary, only the fractional mass $f$ refers to the pair $\mathrm{B}+\mathrm{C}$.

For the four dimensional grid search, we used 100 points each for $f, e$, and $P$. The grid ranged from 0 to 0.99 in $f, 0$ to 0.99 in $e$, and 30 to 300 years in $P$. The grid in $T_{0}$ started with 100 points distributed uniformly over one orbital period. Similar to the fit for the orbit of B-C, the grid in $T_{0}$ was refined until the grid spacing was less than one day.

The results of the grid-search were improved with a Levenberg-Marquardt $\chi^{2}$ minimization algorithm (Press et al. 1992). As starting points, we used all orbits resulting from the grid-search that had $\chi^{2}<\chi_{\min }^{2}+9$. The orbit with the globally minimum $\chi^{2}$ found by the Levenberg-Marquardt fit is shown in Figs. 3 and 4. Table 3 lists its elements, as well as the mass ratio $q$, and the individual masses of all three components. As with the inner orbit of B and C, $\chi^{2}$ is somewhat larger then expected for a good fit.

Errors for the parameters were again estimated by analyzing the $\chi^{2}$ function around the minimum. Since only a relatively small fraction of the orbit has been observed so far, the uncertainties for the orbital elements are much larger than for the orbit of $B$ and $C$ around each other. Figure 5 shows $\chi^{2}$ as function of period and eccentricity. Orbits with periods of more than 100 years are within the $95 \%$ confidence region, and periods of more than 200 years are in the $99.7 \%$ confidence region. The error of the system mass was derived in the same way as with the orbit of LHS $1070 \mathrm{~B}$ and C around each other, i.e. by treating $M$ as an independent fit parameter.

The ambiguity of the ascending node is resolved with the help of Seifahrt et al. (2008). They measured in 2006 a positive relative radial velocity between the barycentre of LHS 1070 B

2 We did not detect the companion D reported by Henry et al. (1999). Therefore, by "A" we mean the suspected close binary composed of A and D (if it exists) in the nomenclature of Henry et al. (1999). 


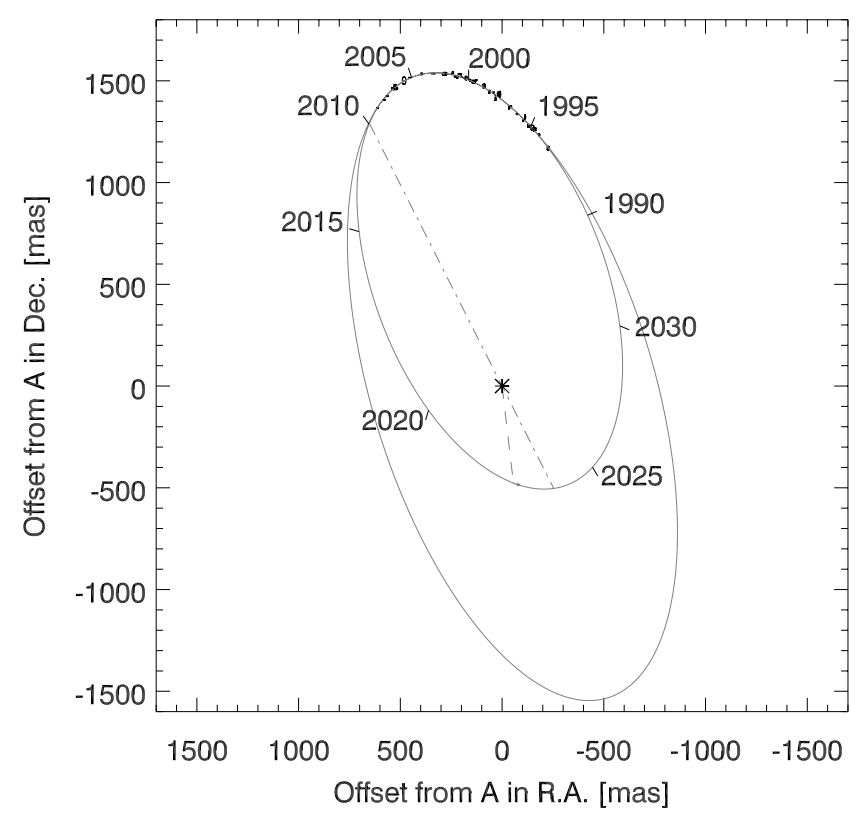

Fig. 3. The orbit of the center of mass of components B and C around component $\mathrm{A}$. The observed positions are marked by their error ellipses and lines connecting the observed and calculated position at the time of the observations. Note that these positions are computed from the observed positions of components $\mathrm{B}$ and $\mathrm{C}$, and the mass ratio $q$, which is a free parameter of the model fit. The dash-dotted line indicates the line of nodes, the dashed line the periastron, and the arrow shows the direction of the orbital motion. The two orbits shown are the model with the globally minimum $\chi^{2}$, and an almost circular orbit with a longer period of about 80 years.

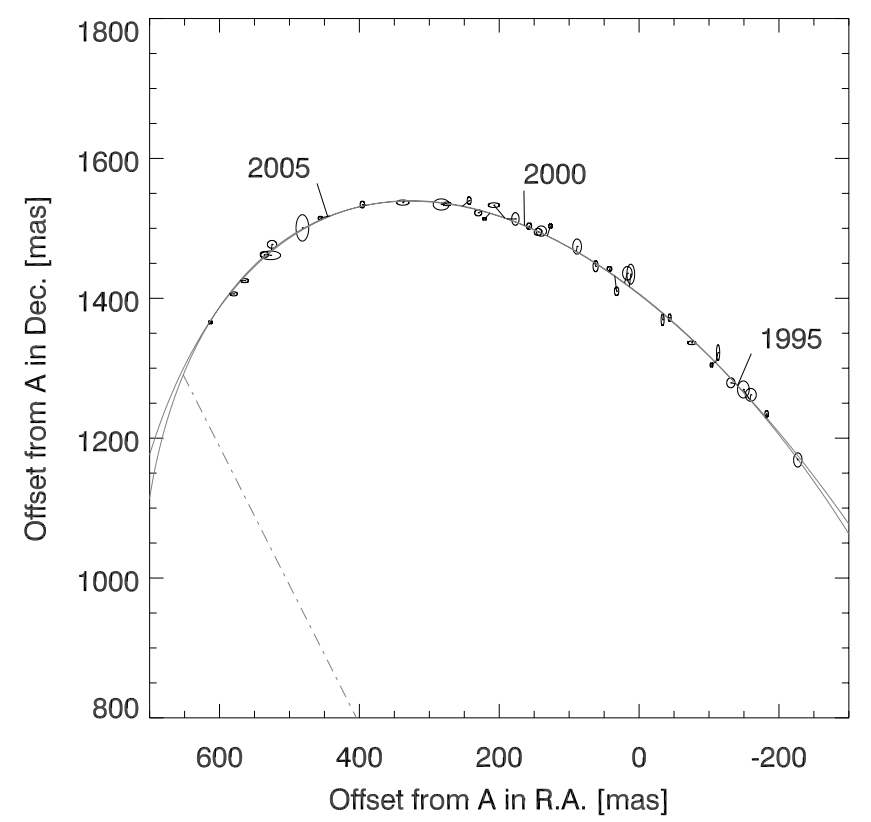

Fig. 4. Enlarged section of the orbits in Fig. 3, showing the part covered by observations.

and $\mathrm{C}$ and component $\mathrm{A}$. Therefore, the binary $\mathrm{BC}$ was receding from the observer, and the position angle of the ascending node must be $26.8^{\circ}$ (cf. Fig. 3). Our orbit model predicts a relative radial velocity of $3.1 \pm 0.1 \mathrm{~km} \mathrm{~s}^{-1}$ on 2006 Oct. 9 , in agreement with the measurement of Seifahrt et al. (2008).

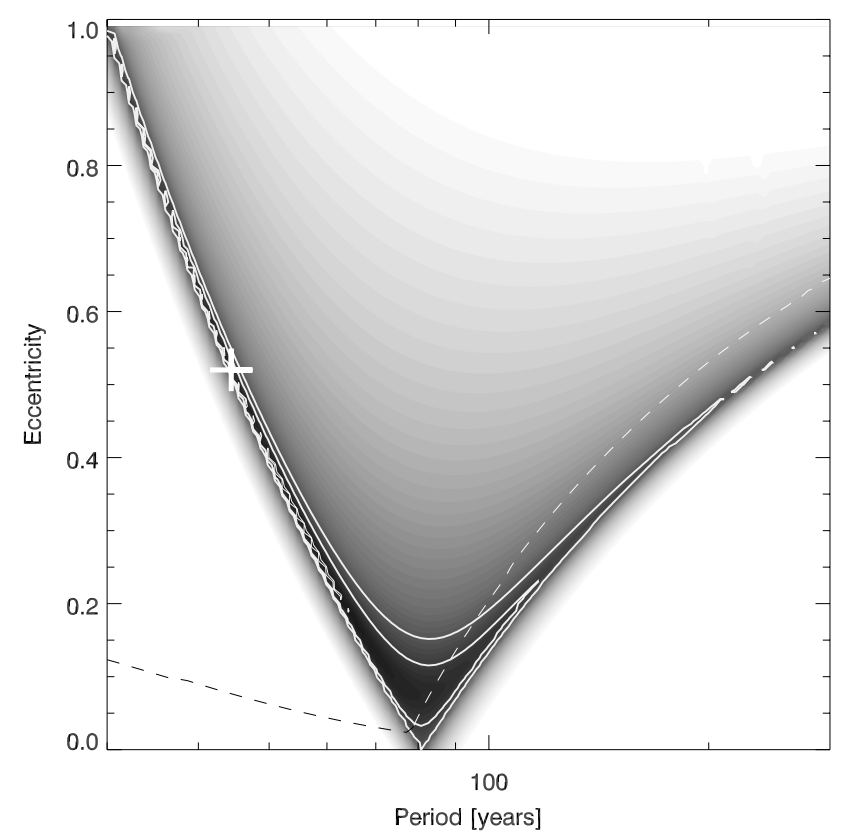

Fig. 5. $\chi^{2}$ as function of $P$ and $e$ for the orbit of $\mathrm{BC}$ around A. The cross at $P=44.4 \mathrm{yr}, e=0.52$ marks the minimum. The contour lines encircle the $68 \%$ confidence region (which is a series of mostly unconnected patches), the $95 \%$, and the $99.7 \%$ confidence region (corresponding to $1 \sigma, 2 \sigma$, and $3 \sigma$ in the case of normally distributed errors). The dashed line marks the stability limit for the system, only orbits below this line are long-term stable (see Sect. 5.1).

Finally, the mass of component A can be computed from the system mass of the triple and the mass of the binary $B+C$ (derived in Sect. 3). The largest contribution to the error of both masses is the uncertainty in the distance to the system. Since this contribution to the errors of $M_{\mathrm{ABC}}$ and $M_{\mathrm{BC}}$ is correlated, it would not be correct to add the errors in quadrature. Instead, we computed masses from the semi-major axes in mas (which results in the unusual mass unit of $\operatorname{mas}^{3} /$ years $^{2}$ ), subtracted $M_{\mathrm{BC}}$ from $M_{\mathrm{ABC}}$, and converted the resulting $M_{\mathrm{A}}$ into solar masses by multiplying it with the distance cubed. This way, the error of the distance enters the calculation only once, resulting in the correct estimate for the error of $M_{\mathrm{A}}$.

\section{Discussion}

\subsection{Is the LHS 1070 system stable?}

Our best fit for the orbit of the binary BC around A is only a factor 2.5 larger than the orbit of BC itself. The outer orbit has also a rather large eccentricity. As a result, the distance from $\mathrm{B}$ or $\mathrm{C}$ to A becomes smaller than the distance to its binary companion during the periastron passage of the outer orbit (Fig. 6). This raises the question whether a triple system like this can be stable over timescales comparable to its age.

Donnison \& Mikulskis (1995) presented a criterion for the long-term stability of coplanar triple systems: The periastron distance $q_{2}=a_{2}\left(1-e_{2}\right)$ of the outer orbit should be at least a factor of 3.3 larger than the semi-major axis $a_{1}$ of the inner orbit. In non-coplanar systems, increasing the inclination decreases the region of stability, i.e. the outer orbit has to be even larger to be stable (Donnison 2009). In Fig. 5, the limit for coplanar orbits $\left(q_{2}>3.3 a_{1}\right)$ is indicated by the dashed line, only orbits below the line are stable. The orbit with the minimum $\chi^{2}$ is clearly in the unstable regime. However, the $2 \sigma$ and $3 \sigma$ confidence regions 


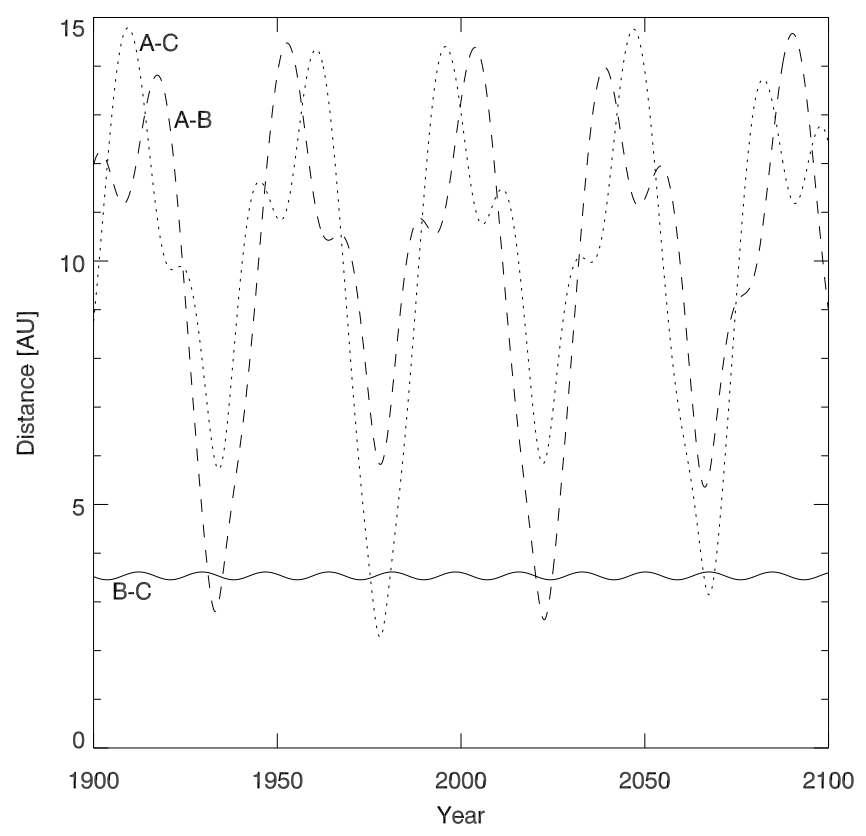

Fig. 6. Distances between the three stars in the system as function of time. Here we assume that the stars stay on the Keplerian orbits derived in Sects. 3 and 4, i.e. we do not simulate the three-body problem posed by the triple system.

extend well into the regime of stable orbits. It is therefore possible that the true orbit has a longer period of at least 80 years (cf. Fig. 3).

How does this uncertainty about the orbit influence our conclusions? Figure 7 shows the parameters of the outer orbit that we are most interested in. Shown are all orbits within the $99 \%$ confidence region in our grid of possible solutions. Unstable orbits (according to the criterion by Donnison \& Mikulskis 1995) are indicated by gray symbols, stable orbits are black. The top panel shows the angle between the planes of the inner and outer orbits. This angle is $12.5^{\circ}$ for the orbit with the minimum $\chi^{2}$, and less than $10^{\circ}$ for all stable orbits. We find a few orbital solutions with angles larger than $100^{\circ}$, but all these orbits are unstable. We therefore conclude that the inner and outer orbit are almost coplanar. Therefore, the stability criterion by Donnison $\&$ Mikulskis (1995) is sufficient in our case, although it does not apply to inclined orbits.

The second panel of Fig. 7 shows the masses of LHS 1070 A and B. Orbits with short periods result in unrealistically high masses for $\mathrm{A}$, which also indicates that the true period is longer. The mass of component B lies between 0.076 and $0.077 M_{\odot}$, almost independent of the orbital period. The mass of component $\mathrm{C}$ was not plotted, since it can easily be computed as $0.149 M_{\odot}-M_{B}$.

Finally, the bottom panel of Fig. 7 shows the mass ratio between LHS $1070 \mathrm{C}$ and B. All stable orbits result in a mass ratio of 0.923 , which is the same as the mass ratio of the orbit with the minimum $\chi^{2}$.

We conclude that it is possible to find stable orbital solutions that are compatible with the astrometric measurements. In these stable configurations, the orbit of $\mathrm{B}$ and $\mathrm{C}$ around each other, and the orbit of $\mathrm{B}+\mathrm{C}$ around $\mathrm{A}$ are almost coplanar, with angles of less than $10^{\circ}$. The mass of component $\mathrm{A}$ lies between 0.13 and $0.16 M_{\odot}$ for stable orbits.

As a final note on the stability of the system, we point out that the stability criterion used here does not take into account

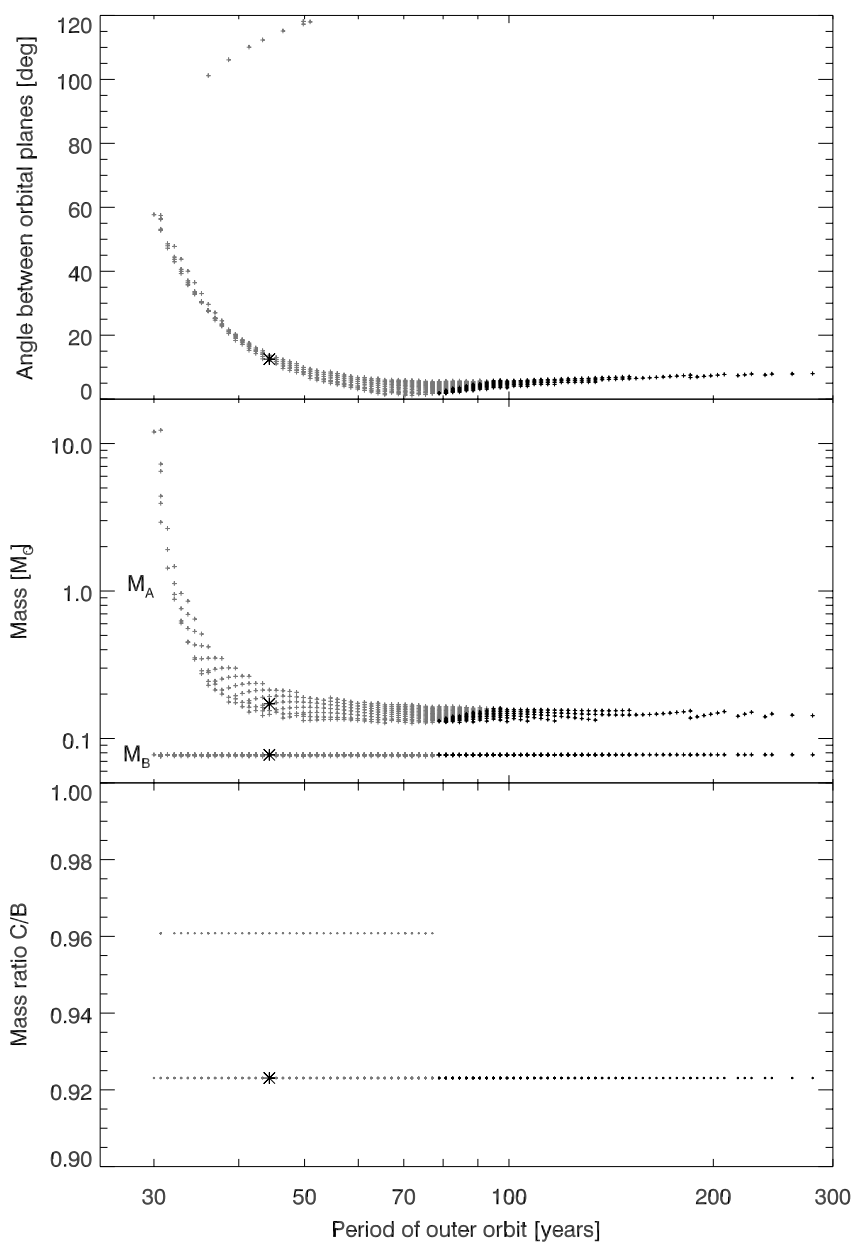

Fig. 7. Results of the fits for the outer orbit as function of their period. Shown are all model orbits within the $99 \%$ confidence region $(3 \sigma)$. Unstable orbits (according to the criterion by Donnison \& Mikulskis 1995) are indicated by gray symbols, stable orbits are black. The orbit with the minimal $\chi^{2}$ is marked by an asterisk.

resonances. For example, a period of the outer orbit of 52 years would be in a 3:1 resonance with the inner orbit. This orbit would be within the $1 \sigma$ region around the orbit model with minimum $\chi^{2}$. However, the uncertainties of our orbit fit are too large to provide a reasonable starting point for a search for stable resonant configurations. Furthermore, it would not significantly change our conclusions about coplanarity and the masses of the three components if the period of the outer orbit was between about 50 and 80 years.

\subsection{Comparison with theoretical models}

The goal of dynamical mass determinations is to test theoretical predictions for the mass and luminosity of the stars. Figure 8 shows a mass-luminosity-diagram with the isochrones of Baraffe et al. (1998) and our results for the components of LHS 1070 (using the $V$-magnitudes given by Leinert et al. 2000). Additionally, it shows the empirical Mass-Luminosity-Relation by Henry et al. (1999), which is similar to the theoretical 500-Myr-isochrone. Within the error bars, both LHS 1070 B and C are compatible with an age between 500 and $800 \mathrm{Myr}$.

On the other hand, LHS $1070 \mathrm{~A}$ is either $3^{\mathrm{mag}}$ too faint or $0.07 M_{\odot}$ too heavy compared to the theoretical models and the empirical relation. This is also true if we take into account that 


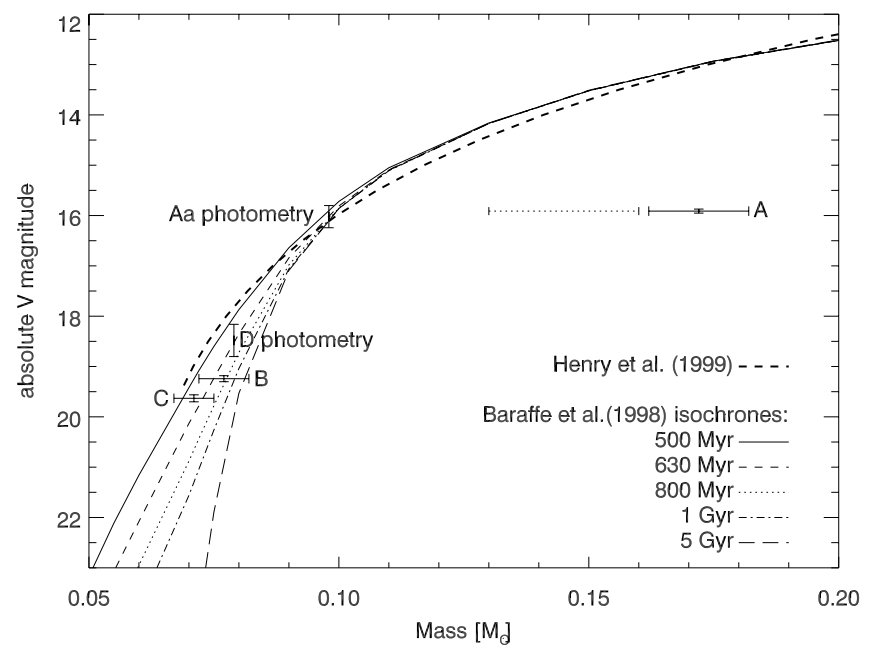

Fig. 8. Mass-luminosity diagram with the components of LHS 1070, the models of Baraffe et al. (1998), and the empirical Mass-LuminosityRelation by Henry et al. (1999). Shown are the theoretical isochrones for ages between $500 \mathrm{Myr}$ and $5 \mathrm{Gyr}$. The dotted horizontal line indicates the mass range for A resulting from stable orbit configurations. The points labeled "Aa photometry" and "D photometry" indicate the magnitudes if A is split into two components (Henry et al. 1999), with the masses adjusted to put the points onto the 630-Myr isochrone.

stable orbit configurations result in lower masses (indicated by the dotted line in Fig. 8). In the $K$-band, LHS $1070 \mathrm{~A}$ is about $1^{\mathrm{mag}}$ too faint compared to the model isochrones. This excludes the possibility that the under-luminosity is due to extinction by circumstellar or foreground material (as unlikely as it may be for a star of this age and distance), since then the effect in the infrared should be smaller, only about $0.3^{\mathrm{mag}}$.

The simplest explanation would be that $\mathrm{A}$ is indeed a binary as reported by Henry et al. (1999). In the following, we will call the components $\mathrm{Aa}$ and $\mathrm{D}$, and continue to use A for the (unresolved) binary system. We computed the absolute $V$-magnitudes of $\mathrm{Aa}$ and $\mathrm{D}$ from the combined apparent magnitude $V=15.35^{\mathrm{mag}}$ (Leinert et al. 2000) and the magnitude difference $\Delta V=2.46^{\mathrm{mag}}$ (Henry et al. 1999), using the distance of $7.72 \mathrm{pc}$ (Costa et al. 2005). By finding the intersection with the 630-Myr isochrone from the models of Baraffe et al. (1998), we can estimate the mass of Aa and D to be $0.10 M_{\odot}$ and $0.08 M_{\odot}$, resp. The combined mass of A resulting from photometry is therefore about $0.18 M_{\odot}$, in reasonable agreement with the dynamical mass resulting from the orbit determinations.

In an attempt to find a possible close companion to LHS $1070 \mathrm{~A}$, we analyzed all available high-resolution data of the system. The resolution required to detect component $\mathrm{D}$ can only be reached with a $8 \mathrm{~m}$-class telescope, preferentially at short wavelengths, e.g. $J$-band. This means we have to rely on NACO data. There are two datasets taken with NACO in the $J$-band on June 27. and December 12., 2003, and two datasets in the narrow-band filter NB_2.17 in a mode that allows speckle postprocessing of the data. In all four datasets, we can exclude an companion with a flux ratio brighter than 0.2 , i.e. a magnitude difference of $1.75^{\mathrm{mag}}$. The resolution of the four observations is 30-40 mas. With the masses of Aa and D derived above, the magnitude difference should be about $0.8^{\mathrm{mag}}$. Assuming a semimajor axis of 50 mas and a system mass of $0.17 M_{\odot}$ results in an orbital period of about 0.6 years. It is possible that all our observations were carried out at times when the companion was too close to the primary to be visible. However, given the large number of attempt to detect LHS 1070 D this appears unlikely.
We also looked into the possibility to detect a periodic astrometric signal of LHS 1070 D in the residuals of the A-BC separation. If LHS $1070 \mathrm{~A}$ is an unresolved binary, then an astrometric shift of its center of light with the period of its orbit can occur. The magnitude of this shift depends on the position of the center of mass and the position of the center of light, hence the mass ratio and the flux ratio. The mass ratio of our suspected Aa-D binary is 0.8 , while the flux ratio computed from the models of Baraffe et al. (1998) is 0.48. The expected motion of the center of light is therefore about 0.12 times the size of the orbit of $\mathrm{D}$ around Aa (6 mas for an orbit of 50 mas). Unfortunately, our sampling is not very dense, with 38 observations in 15.25 years. The Nyquist frequency $v_{\mathrm{N}}=N /(2 T)$ corresponds to a period of 0.8 years. Since our sampling is rather uneven, the Nyquist frequency is not a sharp limit for the detectability of a periodic signal. Nonetheless, due to the sparse sampling, it is unlikely that we can detect periods much shorter than 0.8 years. In fact, neither classical nor generalized Lomb-Scargle-periodograms (Scargle 1982; Zechmeister \& Kürster 2009) show any significant peaks. Even adding an artificial signal with a period of 0.6 years and a large amplitude of 100 mas does not result in a significant detection, although peaks at $1 / 2$ and $1 / 4$ of the frequency appear. In summary, we conclude that an astrometric detection of LHS $1070 \mathrm{D}$ is not possible with the available data.

Another possible explanation could be that LHS 1070 A is a binary too close to be resolved by the observations available so far. This hypothetical companion would not be identical with component D. However, at the moment the most likely explanation might be that we overestimate the system mass. This would not be too surprising, given the small observational coverage of the orbit.

\section{Summary and conclusions}

We present new relative positions of LHS $1070 \mathrm{~A}, \mathrm{~B}$, and C, collected in the years 2000 to 2008. They were used to derive an improved model for the orbit of $\mathrm{B}$ and $\mathrm{C}$ around each other, and an estimate for the orbit of $\mathrm{B}$ and $\mathrm{C}$ around $\mathrm{A}$. The orbit of $\mathrm{B}$ and $\mathrm{C}$ is well-determined by now, with an orbital period of $17.24 \pm 0.01$ years and a system mass of $0.149 \pm 0.009 M_{\odot}$ (cf. Table 2).

The observations of LHS $1070 \mathrm{~B}$ relative to A span only a range in position angle of $36^{\circ}$. The orbit of $\mathrm{B}$ and $\mathrm{C}$ around A derived from these observations is therefore not so wellconstrained. The orbital solution minimizing $\chi^{2}$ has a rather short period of about 44 years, but it results in an unstable configuration of the triple system. It is more likely that the true orbital period is in the range 80-200 years. Orbital solutions in this range of periods are still within the $95 \%$ and $99.7 \%$ confidence region for the fit to the astrometric observations (which corresponds to $2 \sigma$ and $3 \sigma$ for the case of normally distributed measurement errors). Despite this uncertainly, we can constrain the system mass $M_{A}+M_{B}+M_{C}$ to 0.28 to $0.31 M_{\odot}$ if we accept only stable orbit configurations.

The outer orbit also yields the mass ratio of $\mathrm{B}$ and $\mathrm{C}$, which is quite well-constrained to $0.92 \pm 0.01$. Taken together, these results yield individual masses of $M_{A}=0.13 \ldots 0.16 M_{\odot}, M_{B}=$ $0.077 \pm 0.005 M_{\odot}$ and $M_{C}=0.071 \pm 0.004 M_{\odot}$.

Placing the three stars in a mass-luminosity diagram and comparing with theoretical isochrones shows that $\mathrm{B}$ and $\mathrm{C}$ are coeval within the measurement errors, with an age between 500 and $800 \mathrm{Myr}$. On the other hand, LHS $1070 \mathrm{~A}$ appears to be too faint for its mass, or too massive for its luminosity. One possible explanation could be that LHS $1070 \mathrm{~A}$ is itself a binary. 
There has been one report of the discovery of a close companion (Henry et al. 1999), but this discovery could not be confirmed, despite the large number of observations collected. The detection might have been caused by a glitch in the data (Henry, priv. comm.). To confirm or disprove the binarity of LHS $1070 \mathrm{~A}$, observations sensitive to companions at very small separations would be helpful, either spectroscopic or interferometric. For the time being, the most likely explanation might be that our orbit fit overestimates the system mass.

Our results for the masses of the three stars show that LHS $1070 \mathrm{C}$ is almost certainly a brown dwarf. LHS 1070 B is very close to the hydrogen-burning mass-limit, possibly also a brown dwarf. While LHS $1070 \mathrm{~A}$ is clearly above the hydrogenburning limit, it is still a very low mass star. The very low mass triple system LHS 1070 thus has the very interesting property to contain one component above, one below, and one at the hydrogen burning limit. This combination makes it an ideal candidate for testing the change of atmosphere properties in this mass regime. Furthermore, it allows some conclusions about its formation. One theory for the formation of brown dwarfs is that they got ejected out of a multiple system before they could accrete enough mass to start hydrogen burning (Reipurth \& Clarke 2001). It is difficult how a system like LHS 1070 can survive such an event. Even if it managed to remain bound, one would expect more eccentric and inclined orbits than found in this work. It is more likely that LHS 1070 formed in the same way as normal stars, where a number of triple and higher-order multiple systems are known.

Acknowledgements. We thank the many observers and support astronomers who carried out the many separate observations necessary for this work. We are also grateful for the helpful comments by the editor.

\section{References}

Almeida, L. A., Jablonski, F., \& Martioli, E. 2011, A\&A, 525, A84

Baraffe, I., Chabrier, G., Allard, F., \& Hauschildt, P. H. 1998, A\&A, 337, 403
Basri, G., \& Marcy, G. W. 1995, AJ, 109, 762

Costa, E., Méndez, R. A., Jao, W.-C., et al. 2005, AJ, 130, 337

Diolaiti, E., Bendinelli, O., Bonaccini, D., et al. 2000, A\&AS, 147, 335

Donnison, J. R. 2009, Planet. Space Sci., 57, 771

Donnison, J. R., \& Mikulskis, D. F. 1995, MNRAS, 272, 1

Heintz, W. D. 1978, Double Stars, Geophysics and Astrophysics Monographs No. 15 (Dordrecht, Holland: D. Reidel Publishing Company)

Henry, T. J., Franz, O. G., Wasserman, L. H., et al. 1999, ApJ, 512, 864

Hofmann, R., Blietz, M., Duhoux, P., et al. 1992, in Progress in Telescope and Instrumentation Technologies, ESO Conference and Workshop Proc. No. 42, ed. M.-H. Ulrich (ESO Garching), 617

Hofmann, R., Brandl, B., Eckart, A., Eisenhauer, F., \& Tacconi-Garman, L. E. 1995, in SPIE 2475, ed. A. M. Fowler, 192

Köhler, R., Kunkel, M., Leinert, C., \& Zinnecker, H. 2000, A\&A, 356, 541

Köhler, R., Ratzka, T., Herbst, T. M., \& Kasper, M. 2008, A\&A, 482, 929

Leinert, C., Weitzel, N., Richichi, A., Eckart, A., \& Tacconi-Garman, L. E. 1994, A\&A, 291, L47

Leinert, C., Allard, F., Richichi, A., \& Hauschildt, P. H. 2000, A\&A, 353, 691

Leinert, C., Jahreiß, H., Woitas, J., et al. 2001, A\&A, 367, 183

Lenzen, R., Bizenberger, P., Salm, N., \& Storz, C. 1998, in SPIE Conf. Ser. 3354, ed. A. M. Fowler, 493

Lenzen, R., Hartung, M., Brandner, W., et al. 2003, in Instrument Design and Performance for Optical/Infrared Ground-based Telescopes, ed. M. Iye, \& A. F. M. Moorwood, SPIE Proc., 4841, 944

McCaughrean, M. J., \& Stauffer, J. R. 1994, AJ, 108, 1382

Press, W. H., Teukolsky, S. A., Vetterling, W. T., \& Flannery, B. P. 1992, Numerical Recipes in C, 2nd edn. (Cambridge, UK: Cambridge University Press)

Reiners, A., Seifahrt, A., Käufl, H. U., Siebenmorgen, R., \& Smette, A. 2007, A\&A, 471, L5

Reipurth, B., \& Clarke, C. 2001, AJ, 122, 432

Rousset, G., \& Beuzit, J.-L. 1999, The COME-ON/ADONIS systems, ed. F. Roddier, 171

Rousset, G., Lacombe, F., Puget, P., et al. 2003, in Adaptive Optical System Technologies II, ed. P. L. Wizinowich, \& D. Bonaccini, SPIE Proc. 4839, 140

Scargle, J. D. 1982, ApJ, 263, 835

Seifahrt, A., Röll, T., Neuhäuser, R., et al. 2008, A\&A, 484, 429

van Altena, W. F., Lee, J. T., \& Hoffleit, E. D. 1995, The general catalogue of trigonometric [stellar] parallaxes, ed. W. F. van Altena, J. T. Lee, \& E. D. Hoffleit

Zechmeister, M., \& Kürster, M. 2009, A\&A, 496, 577 